\title{
Numerical Simulations of MHD Accretion Disks
}

\author{
J. F. Hawley \\ Department of Astronomy, University of Virginia, Charlottesville, VA 22904, USA \\ jh8h@virginia.edu
}

\begin{abstract}
Numerical simulations play an increasingly important role in investigating accretion disks and associated phenomena such as jets. This paper provides a few examples of recent results that have been obtained with simulations, both local or global.
\end{abstract}

Keywords. accretion, accretion disks, magnetohydrodynamics: MHD

The most energetic phenomena in the universe are systems powered by gravity through accretion, specifically accretion disks surrounding compact stars and black holes. Accretion theory has largely been based primarily on a one-dimensional time-steady model consisting an optically thick, vertically-thin, Keplerian disk with an unknown, parameterized internal stress. While analytic models of this type provide considerable insight, their limitations are now well-known, and the observational data demand moving beyond this standard. Numerical simulations provide another means for investigating accretion disks.

The governing equations of accretion are those of compressible magnetohydrodynamics (MHD). Magnetic fields render a differentially rotating fluid unstable to the magnetorotational instability - MRI; Balbus \& Hawley (1991) - and the resulting turbulence accounts for the internal stress that drives accretion. These processes have been studied using local "shearing box" simulations - Hawley, Gammie \& Balbus (1995). The shearing box is a Cartesian domain representing a small piece of the disk, assumed to be rotating with the local disk angular velocity, $\Omega$. The equations include differential rotation, Coriolis force and the tidal potential. Local simulations have shown how the MRI produces angular momentum transfer and the degree to which the MRI stress acts like a Shakura-Sunyaev " $\alpha$ viscosity," where $\tau_{r \phi}=\alpha P$ (Balbus \& Papaloizou (1999)). MRI-driven turbulence generates substantial local stress, typically with $\alpha \sim 0.01$. The orbital energy released by that stress is locally dissipated by the turbulence into heat in an eddy turnover time (Simon, Hawley, \& Beckwith (2009)). Shearing box simulations that combine MHD with flux limited diffusion (Hirose, Krolik, \& Blaes (2009)) find that while the stress is roughly proportional to the total pressure (including both gas and radiation), the stress determines the pressure, not other way around. Increases in the stress lead to stronger turbulence, more turbulent dissipation, and more heat. A consequence of this is that radiation pressure-supported accretion disks are thermally stable, in contrast to expectations based on assuming a strict $\alpha$ viscosity. Because of its fundamentally magnetic nature, the stress is directly proportional to the magnetic energy which is itself determined by the balance between MRI driving (at a range of scales) and turbulent dissipation. At the present time, however, we don't have a way to predict the magnetic field energy as a local function of the state of the disk.

The recognition that MHD turbulence provides the stress in disks leads to new questions. For example, because magnetic stresses don't necessarily diminish in the plunging region inside the inner most stable circular orbit (ISCO) around a black hole, the overall efficiency and luminosity of disks can be greater than predicted by models that assume 
"zero torque" at the ISCO. Also, the magnetic field embedded in the accretion flow can be brought into a rotating black hole's ergosphere, possibly to form a relativistic jet. Issues such as these must be investigated through global simulations of disks around rotating black holes.

De Villiers, Hawley \& Krolik (2003) carried out some of the first long-term, threedimensional MHD global disk simulations for a variety of black hole spins. These simulations, and others that followed, have shown that significant stress can continue at and inside the ISCO and all the way to the horizon for rotating black holes. The most significant unanswered question is what factors (e.g., disk thickness, thermal pressure, magnetic topology) determine the level of the stress at the ISCO. Shafee et al. (2008) recently simulated a thin disk around a Schwarzschild hole and suggested that the ISCO stress cuts off sharply when the disk is thin. On the other hand, Noble, Krolik, \& Hawley (2009) find additional ISCO stress even for a relatively thin disk. They further considered the observational implications of the ISCO stress using simple emission and absorption models coupled with relativistic ray tracing. Work continues on this important issue.

Simulations have produced new insights into jet production. Under the right circumstances, reasonably long-lived large-scale field can be brought down and attached to the black hole. Field loops originally contained entirely within the accretion flow can expand dramatically within a cone around the rotation axis, successfully supporting jets carrying substantial Poynting flux to large distance (Hawley \& Krolik (2006)) if the black hole is rotating. However, the longevity and strength of these large-scale fields - and their associated jets - depend critically on the topology of those loops (Beckwith, Hawley \& Krolik (2008)). A strong, long-lived jet seems to require a net vertical field in the disk midplane whose sign remains consistent for at least an inner-disk inflow time.

In conclusion, although the time- and length-scales involved make accretion disk simulations challenging, they have revealed new details about time-dependent properties of disks, magnetic disk dynamos, jet launching mechanisms, and the dynamical properties of systems other than the standard thin disk. As the capabilities of computational hardware increase, and the development of advanced numerical codes continues, our theoretical understanding of accretion physics should substantially improve.

\section{Acknowledgements}

This work was supported by NASA grant NNX09AD14G. I thank collaborators Kris Beckwith, Jean-Pierre De Villiers, Andrew Hamilton, Julian Krolik, Scott Noble, Jeremy Schnittman, and Jacob Simon. Computational resources were supplied by the TeraGrid, supported by the National Science Foundation.

\section{References}

Balbus, S. A. \& Hawley, J. F. 1991, ApJ, 376, 214

Balbus, S. A. \& Papaloizou, J. C. B. 1999, ApJ, 521, 650

Beckwith, K., Hawley, J. F., \& Krolik, J. H. 2008, ApJ, 678, 1180

De Villiers, J.-P., Hawley, J. F., \& Krolik, J. H. 2003, ApJ, 599, 1238

Hawley, J. F, Gammie, C. F., \& Balbus S. A. 1995, ApJ, 440, 742

Hawley, J. F. \& Krolik, J. H. 2006, ApJ, 641, 103

Hirose, S., Krolik, J. H., \& Blaes, O. 2009, ApJ, 691, 16

Noble, S. C., Krolik, J. H., \& Hawley, J. F. 2009, ApJ, 692, 411

Shafee, R. et al. 2008, ApJ, 687, L25

Simon, J. B., Hawley, J. F., \& Beckwith, K. 2009, ApJ, 690, 974 